\title{
Self-consistent coupling of radiative transfer and dynamics in dust driven winds
}

\author{
Nicole Berruyer \\ Observatoire de la Côte d'Azur, Laboratoire G.-D. Cassini, \\ CNRS/UMR 6529, BP 4299, F-06304 Nice, Cedex 4, la France \\ Jean-Pierre J. Lafon \\ Observatoire de Paris-Meudon, GEPI, F-92195 Meudon, la France
}

\author{
Stéphane Liberatore \\ Commissariat à l'Energie Atomique, DPTA, \\ F-91680 Bruyères-Le-Châtel, la France
}

\begin{abstract}
The aim is to determine the role and influence of the hypothesis concerning both dynamics and radiative transfer in models of winds and mass loss of evolved stars, when the radiative force on dust grains plays a major role in the structuration of the circumstellar envelope of the star. The flow is described successively using two models coupling the grains-gas dynamics in a self-consistent way with radiative transfer for two different approaches of the dynamics: the Momentum Coupling Hypothesis and the Full Problem. Complete radiative transfer including multiple scattering, absorption and thermal emission is taken into account. The medium is not necessarily optically thin. In all cases, numerical iterations couple dynamics with transfer. This study emphasizes the importance of the drift velocity between the grains and the gas and the inertia of dust together with hydrodynamics and transfer coupling.
\end{abstract}

\section{Introduction}

The existence of circumstellar dust grains and mass loss in cool stars is by now well established. Whatever the mechanisms causing the wind, the mass loss is controlled by the radiative pressure on dust grains. Photons emitted by the star are absorbed by grains and partially scattered in a more or less isotropic way. A part of the momentum carried by the absorbed photons is transferred to the grains. Friction between grains and atoms or molecules then transmits part of the grain's momentum to the gas.

\section{Wind models and results}

The following simplifying assumptions are used: The flow is stationary, spherically symmetric and grains exist above some reference level. The wind is neutral and isothermal. The effects in anisotropic scattering are taken into account.

In the Full problem (FPB) the flow is described by a set of two differential equations, one for the grains and one for the gas, coupled by collisional drag 

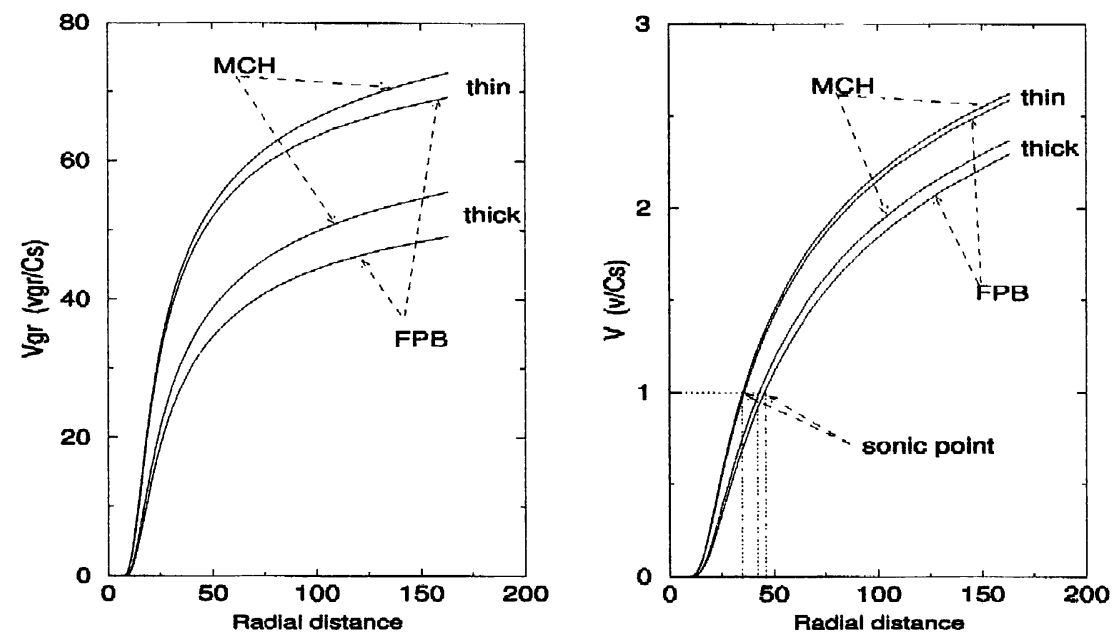

Figure 1. Velocity profiles of dust grain $\left(v_{g r}\right)$ and gas $(v) v s$. the radial distance (in units of the star radius: $R=r / r_{*}$ ). MCH and FPB are compared for the optically thin and thick solutions.

(Berruyer \& Frisch 1983). In the Momentum Coupling Hypothesis (MCH) gas and grains are also coupled by friction, but the whole momentum given to grains by the radiative force is transferred to the gas by grain-gas collisions.

In each case, dynamics is self-consitently coupled with a radiative transfer model based on the 'Quasi-Diffusion Method' developed by Leung (1975). For more details about our wind models see Liberatore et al. (2001).

The input physical parameters for the two models are: $R_{*}=253 \mathrm{R}_{\odot}, L_{*}=$ $4.6 \times 10^{3} \mathrm{~L}_{\odot}, M_{*}=1 \mathrm{M}_{\odot}$ (stellar radius, luminosity and mass),$T_{*}=3000 \mathrm{~K}$, $T_{\text {gas }}=1300 \mathrm{~K}$ (star and gas temperature), $R_{\mathrm{i}}=8.15 R_{*}, R_{\mathrm{o}}=163 R_{*}$ (inner and outer radius of the envelope), $\dot{M}=0.45 \times 10^{-7} \mathrm{M}_{\odot} \mathrm{yr}^{-1}$ and $\dot{M}_{\mathrm{gr}}=0.9 \times 10^{-10}$ $\mathrm{M}_{\odot} \mathrm{yr}^{-1}$ (mass loss rate of gas and dust grains).

In the Figure 1, 'thin' models (for MCH and FPB) are compared with 'thick' models (where the medium is optically thick). We see that differences between velocities increase. Thus the first conclusion is that with a self-consistent treatment, dynamics requires the solution of the full problem. With the self-consistent model, the decrease of $v_{\mathrm{gr}}$ is faster that the decrease of $v$, so that the drift velocity $v_{\mathrm{d}}=v_{\mathrm{gr}}-v$ decreases. Finally, the effect of the radiative transfer on the drift velocity $v_{\mathrm{d}}$ described for $\mathrm{MCH}$ is amplified under FPB conditions. The gas is less sensitive than the dust grains to variations of physical parameters because it is a fluid.

\section{References}

Berruyer, N., Frisch, H. 1983, A\&A 126, 269

Leung, C.M. 1975, ApJ 199, 340

Liberatore, S., Lafon, J.P.J., Berruyer, N. 2001, A\&A 377, 522 